\title{
Aneuploidy as a mechanism for stress-induced liver adaptation
}

\author{
Andrew W. Duncan, ${ }^{1}$ Amy E. Hanlon Newell, ${ }^{2}$ Weimin $\mathrm{Bi},{ }^{3}$ \\ Milton J. Finegold, ${ }^{4}$ Susan B. Olson, ${ }^{2}$ Arthur L. Beaudet, ${ }^{3}$ and Markus Grompe ${ }^{1}$ \\ 10regon Stem Cell Center, Papé Family Pediatric Research Institute, and 2Department of Molecular and Medical Genetics, \\ Oregon Health and Science University, Portland, Oregon, USA. ${ }^{3}$ Department of Molecular and Human Genetics, \\ Baylor College of Medicine, Houston, Texas, USA. ${ }^{4}$ Department of Pathology, Texas Children's Hospital, Houston, Texas, USA.
}

\begin{abstract}
Over half of the mature hepatocytes in mice and humans are aneuploid and yet retain full ability to undergo mitosis. This observation has raised the question of whether this unusual somatic genetic variation evolved as an adaptive mechanism in response to hepatic injury. According to this model, hepatotoxic insults select for hepatocytes with specific numerical chromosome abnormalities, rendering them differentially resistant to injury. To test this hypothesis, we utilized a strain of mice heterozygous for a mutation in the homogentisic acid dioxygenase $(H g d)$ gene located on chromosome 16 . Loss of the remaining $H g d$ allele protects from fumarylacetoacetate hydrolase $(F a b)$ deficiency, a genetic liver disease model. When adult mice heterozygous for $\mathrm{Hgd}$ and lacking $\mathrm{Fab}$ were exposed to chronic liver damage, injury-resistant nodules consisting of $\mathrm{Hgd}$ null hepatocytes rapidly emerged. To determine whether aneuploidy played a role in this phenomenon, array comparative genomic hybridization (aCGH) and metaphase karyotyping were performed. Strikingly, loss of chromosome 16 was dramatically enriched in all mice that became completely resistant to tyrosinemiainduced hepatic injury. The frequency of chromosome 16-specific aneuploidy was approximately $50 \%$. This result indicates that selection of a specific aneuploid karyotype can result in the adaptation of hepatocytes to chronic liver injury. The extent to which aneuploidy promotes hepatic adaptation in humans remains under investigation.
\end{abstract}

\section{Introduction}

Since all portal blood first passes through the liver, this organ is continuously exposed to nutritional challenges as well as xenobiotic insults (reviewed in refs. 1,2). As a result, the liver has developed an amazing capacity for regeneration. Rats can survive up to 7 sequential $50 \%$ hepatectomies, demonstrating a virtually inexhaustible replicative capacity (3). Similarly, serial transplantation of mature hepatocytes in mice indicates their stem cell-like regenerative capacity (4).

In addition to the well-known regenerative capacity of the liver, polyploidy in the liver is extensively described in many vertebrate species, including humans $(5,6)$, rats $(7)$, and mice $(8,9)$. Polyploidization usually occurs by failed cytokinesis $(7,10)$, but cell fusion can also give rise to polyploid cells (11). Although polyploid hepatocytes were historically thought to have limited mitotic capacity, we $(12,13)$ and others $(14)$ have shown that tetraploid and octaploid mouse hepatocytes are highly regenerative. Furthermore, we showed that polyploid hepatocytes could undergo reductive divisions and generate diploid daughters $(12,15)$ both in vitro and in vivo (11). We termed this phenomenon the "ploidy conveyor." When polyploid hepatocytes divide, multiple mitotic spindles are initially established. In most cases, these multiple spindles resolve in a single bipolar spindle, but multipolar mitoses also occur (12). Tripolar mitoses and double mitoses can produce 3 and 4 daughters with reduced ploidy in a single division. These daughters are frequently highly aneuploid, displaying multiple chromosome gains and losses. However, even the bipolar mitoses of polyploid hepatocytes often result in chromosome

Conflict of interest: The authors have declared that no conflict of interest exists. Citation for this article: J Clin Invest. 2012;122(9):3307-3315. doi:10.1172/JCI64026. missegregation due to sorting errors caused by merotelic chromosome attachments (16). Since most hepatocytes become polyploid in the postnatal period when growth and regeneration are ongoing, the overall result is that most hepatocytes of adult mice are aneuploid (12). Recently, we found that this is true for healthy human liver as well (17).

The observed hepatocyte aneuploidy appears random in healthy individuals, and gains and losses of whole chromosomes are seen. Extensive genetic heterogeneity is often beneficial in terms of providing a substrate for selection of the fittest phenotypes. While this concept has been eloquently demonstrated in yeast $(18,19)$, we wondered whether the liver could also utilize this mechanism to adapt to chronic injury. Gene expression is dosage sensitive, and chromosomes harboring susceptibility or resistance alleles for a particular injury could be selected for or against.

To test this hypothesis, we utilized a genetic liver disease model, hereditary tyrosinemia type I, caused by deficiency of fumarylacetoacetate hydrolase (FAH) (ref. 20 and Figure 1A). We (21) and others (22) have previously shown that loss of enzymes upstream of FAH can be protective and prevent hereditary tyrosinemia type I. In fact, treatment of this disorder is based on pharmacological inhibition of hydroxy-phenylpyruvate dioxygenase (HPD) using the drug 2-(2-nitro-4-trifluoro- methylbenzoyl)-1,3-cyclohexanedione (NTBC). Mice deficient in FAH, but heterozygous for a mutation in homogentisic acid dioxygenase (HGD), can develop revertant, healthy hepatocyte nodules that have lost the WT copy of $H g d$ (21). Although $25 \%$ of the reversions are due to point mutations of the WT Hgd locus (21), complete gene loss represents most of the events. Here, we demonstrate that adaptive aneuploidy with nonrandom loss of chromosome 16 accounts for the majority of the injury-resistant hepatocytes. 
A

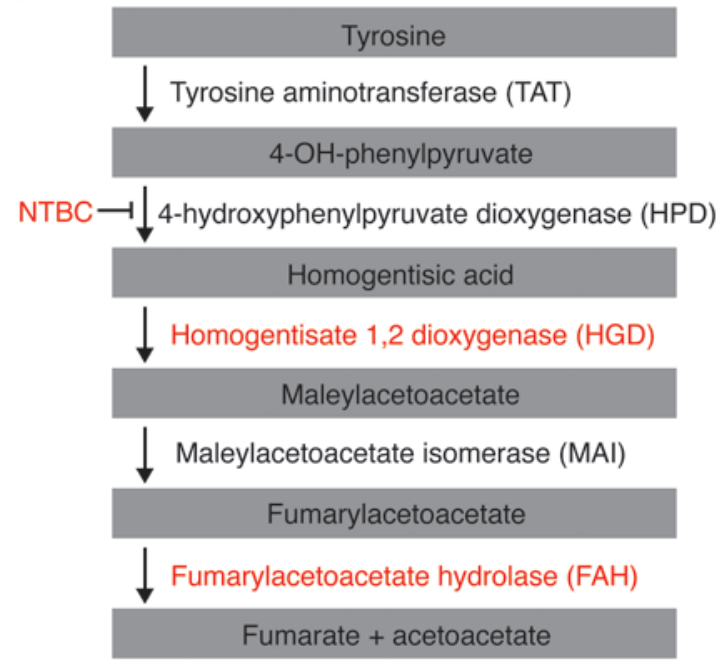

E

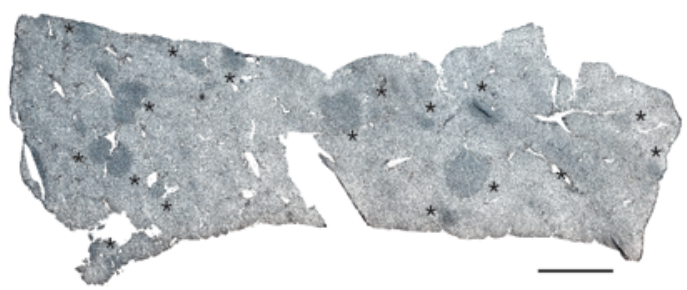

B

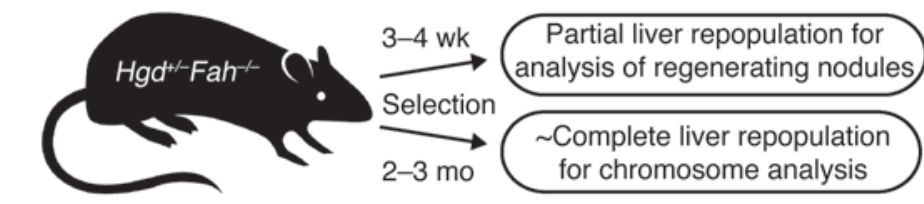

C

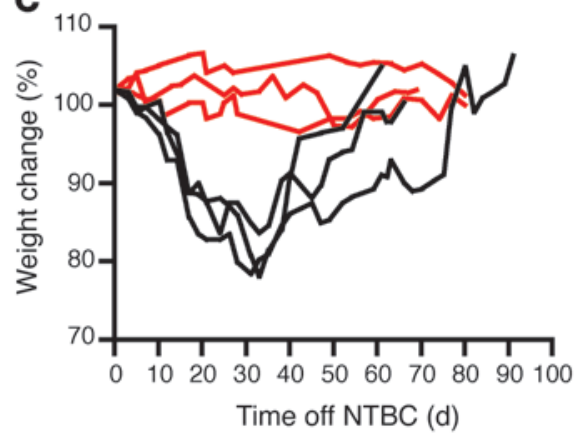

D

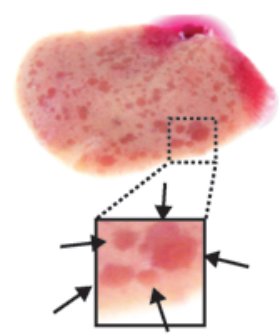

$\mathbf{F}$
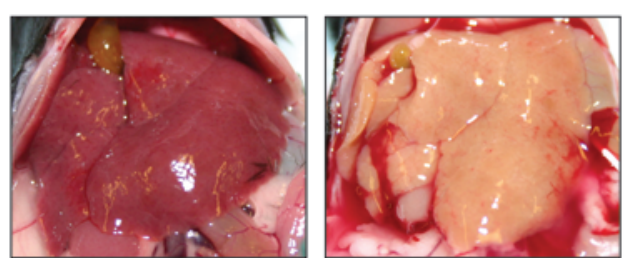

\section{Figure 1}

Spontaneous liver repopulation in response to liver injury. (A) Diagram of the tyrosine catabolic pathway. Critical components are highlighted in red. Fah deficiency leads to accumulation of fumarylacetoacetate and toxic metabolites. Fah deficiency is ameliorated by NTBC therapy or loss of $\mathrm{Hgd}$. (B) $\mathrm{Hgd}^{+/-} \mathrm{Fah}^{-/-}$mice were subjected to selection by NTBC withdrawal and regenerating livers analyzed following partial and approximately complete repopulation. (C) During NTBC selection, $\mathrm{Hgd}^{+/-} \mathrm{Fah}{ }^{-/-}$mice (black lines; $n=3$ ) lost approximately $20 \%$ of their starting weight over

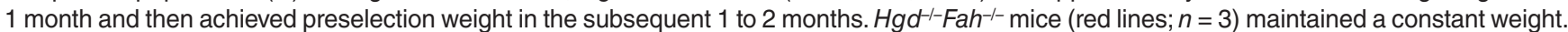
(D and E) Following 3 to 4 weeks of selection, hepatic nodules were dispersed randomly throughout livers from $\mathrm{Hgd}^{+/-} \mathrm{Fah}^{-/-}$mice. Nodules maintained a healthy appearance (D, arrows, inset is enlarged 250\%) $(n=18)$ and were positive for the proliferation marker Ki67 (E, asterisks) $(n=6)$. Scale bar: $1 \mathrm{~mm}$. (F) Following approximately complete repopulation, livers had normal coloration (left panel) and were confluent with large nodules $(n=5)$, which are best observed macroscopically after exsanguination (right panel).

\section{Results}

Proliferation by a subset of hepatocytes in $\mathrm{Hgd}^{+/-} \mathrm{Fah}^{-/-}$mice. To investigate the mechanism of stress-induced hepatocyte proliferation, we utilized mice with mutations in the tyrosine catabolic pathway. Mice heterozygous for $\mathrm{Hgd}$ and deficient for Fab were maintained with an NTBC-supplemented diet, which allows these mutants to survive and maintain normal liver function (Figure $1 \mathrm{~A}$ and ref. 23). NTBC was removed for defined periods beginning at age 3 to 4 months (Figure 1B). After 3 to 4 weeks of selection (i.e., without NTBC-supplemented diet), body weight was reduced by approximately $20 \%$ and animals appeared sickly (Figure 1C). In contrast, $\mathrm{Hgd}^{-/-} \mathrm{Fab}^{-/-}$controls looked healthy and maintained constant body weight. Although the majority of liver mass was pale, which is similar to tissue from $\mathrm{Fab}^{-/-}$mice, $\mathrm{Hgd}^{+/-} \mathrm{Fab}^{-/-}$livers were decorated with red healthy looking hepatic nodules dispersed throughout each lobe (Figure 1D). Consistent with previous results (21), livers from these mice were enriched for discrete regions of $\mathrm{Ki}^{+} 7^{+}$ hepatocytes, indicating proliferation by clonal hepatic populations (Figure 1E). Additionally, 5\%-10\% of these nodules were associated with loss of genetic and chromosome markers, suggesting clonal aneuploidy within the nodules (Supplemental
Figure 1; supplemental material available online with this article; doi:10.1172/JCI64026DS1). Following 2 to 3 months of selection, approximately $10 \%$ of $\mathrm{Hgd}^{+/-} \mathrm{Fah}^{-/-}$mice regained normal body weight (Figure 1C) and livers were repopulated $75 \%$ or more with healthy tissue (Figure 1F). Moreover, in transplantation experiments, hepatocytes derived from injury-resistant $\mathrm{Hgd}^{+/-} \mathrm{Fab}^{-/-}$livers underwent extensive in vivo proliferation and restored liver function in $\mathrm{Fah}^{-/-}$recipients (data not shown).

Together, the data indicate that liver repopulation in $\mathrm{Hgd}^{+/-} \mathrm{Fab}^{-/-}$ mice proceeds in a step-wise manner in response to tyrosinemia. First, the uninjured liver contains a subset of hepatocytes, which have the capacity for functional adaptation. Such cells are randomly dispersed throughout the liver. Secondly, following chronic liver injury or stress (e.g., removal of NTBC) adapted hepatocytes maintain a functional and proliferative advantage, generating healthy nodules after several weeks. Finally, in response to continued hepatic injury, injury-resistant nodules continue to proliferate and restore the entire liver mass.

Chromosome 16 is lost by injury-resistant livers. Our group previously showed that approximately $25 \%$ of regenerating hepatic nodules in $\mathrm{Hgd}^{+/-} \mathrm{Fah}^{-/-}$mice acquired point mutations within the WT Hgd 
A

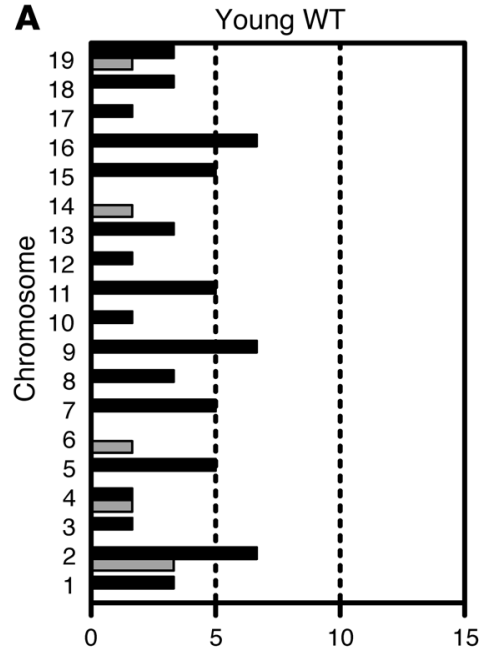

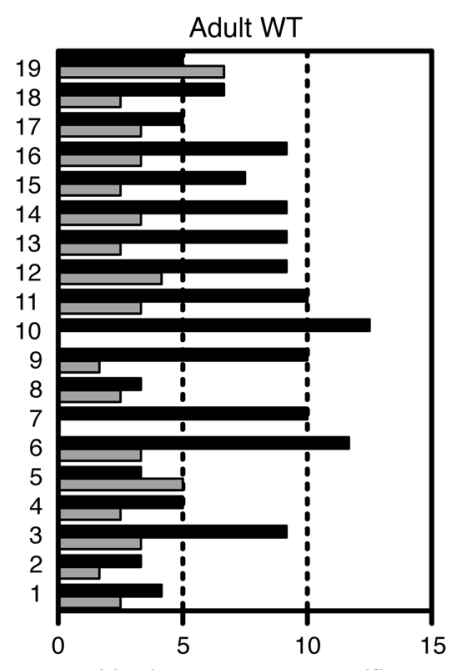

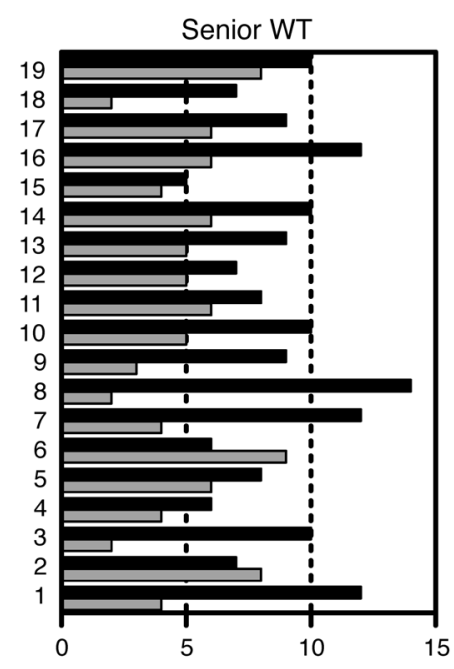

B

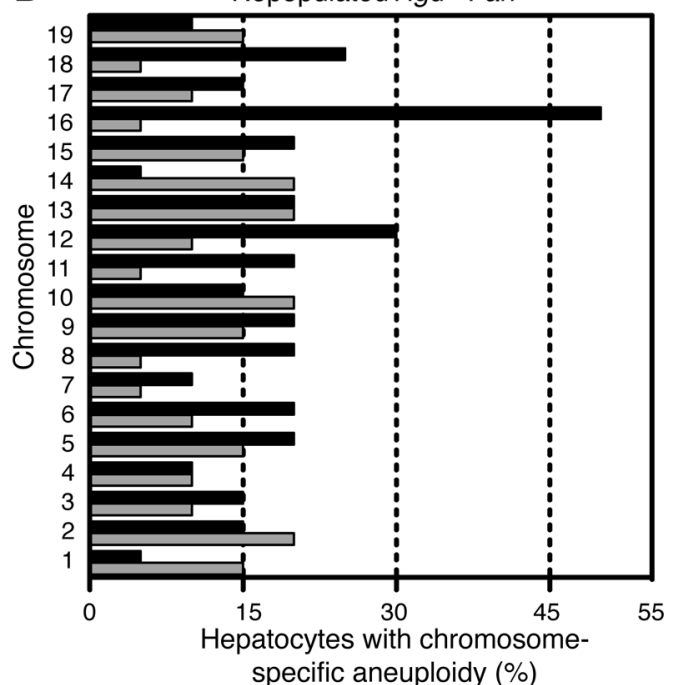

C

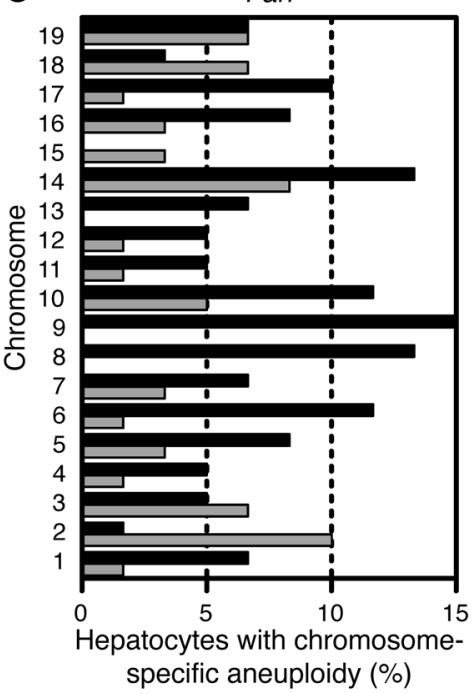

D

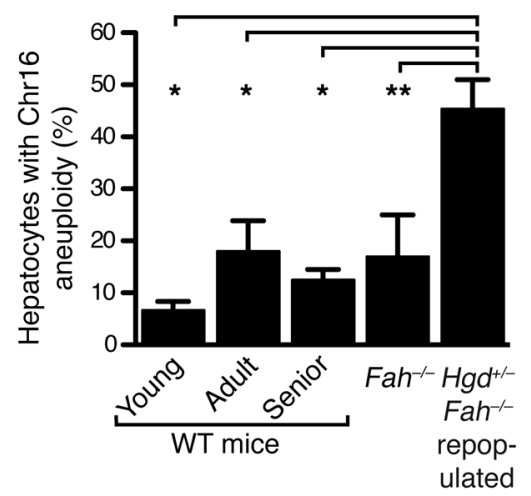

Figure 2

Whole chromosome aneuploidy in hepatocytes. (A) Hepatocytes isolated from healthy WT mice were karyotyped. The percentages of hepatocytes with chromosome-specific gains (gray bars) and losses (black bars) are indicated for young mice (ages 20 to 21 days; $n=3$ ), adult mice (ages 4 to 5 months; $n=5$ ), and senior mice (ages 10 to 15 months; $n=6$ ). Percentages were derived from pooled data within each group. (B) Hepatocytes from fully repopulated $\mathrm{Hgd}^{+/-} \mathrm{Fah}^{-/-}$mice were karyotyped $(n=4)$; a representative profile is shown. (C) Karyotypes were also determined for hepatocytes from Fah ${ }^{-1-}$ mice that were off NTBC. Percentages were derived from pooled data $(n=4)$. (D) Chromosome 16 aneuploidy is indicated for all mice karyotyped. Data are shown as mean \pm SEM. ${ }^{*} P<0.005 ;{ }^{* \star} P=0.03$.

gene, leading to loss of function (21). Lack of functional HGD therefore blocked tyrosine catabolism at the level of homogentisic acid (Figure 1A), allowing these hepatocytes to survive and proliferate during selection. Based on the high degree of aneuploidy in the murine liver (12), we hypothesized that hepatic aneuploidy could contribute to the adaptive response by $\mathrm{Hgd}^{+/-} \mathrm{Fab}^{-/-}$mice. The genomic sequence encoding $H g d$ is located centrally in the qB3 region of chromosome 16. As Hgd heterozygotes have a single functional copy of $\mathrm{Hgd}$ per diploid genome, aneuploid hepatocytes lacking chromosome 16 with the functional gene product would be completely devoid of HGD activity.

To test whether chromosome 16 loss contributed to liver adaptation, we first determined the baseline degree of aneuploidy by karyotyping hepatocytes from different ages of WT mice (Figure 2A).
Females and males were included in this survey, so the analysis was restricted to autosomes only. Gains of each individual autosome were detected in hepatocytes from every age group: young 20 - to 21 -day-old mice $(0.6 \%$, range $0 \%-3 \%)$, adult 4 - to 5 -monthold mice $(3 \%$, range $0 \%-7 \%)$, and senior 10 - to 15 -month-old mice $(5 \%$, range $2 \%-9 \%)$. Similarly, losses of each autosome were also detected in hepatocytes from every group: young mice $(3 \%$, range $0 \%-7 \%$ ), adults $(8 \%$, range $3 \%-12 \%)$, and seniors $(9 \%$, range $5 \%-14 \%$ ). In adults, for example, gain of chromosome 3 was seen in $3 \%$ of hepatocytes, whereas chromosome 3 loss was observed in $9 \%$ of hepatocytes. Next, we karyotyped hepatocytes from $\mathrm{Hgd}^{+/-} \mathrm{Fah}^{-/-}$mice that were highly repopulated ( $\left.275 \%\right)$ after NTBC withdrawal and resistant to injury. Aneuploidy was detected for each autosome. Compared with WT hepatocytes, the frequency of 


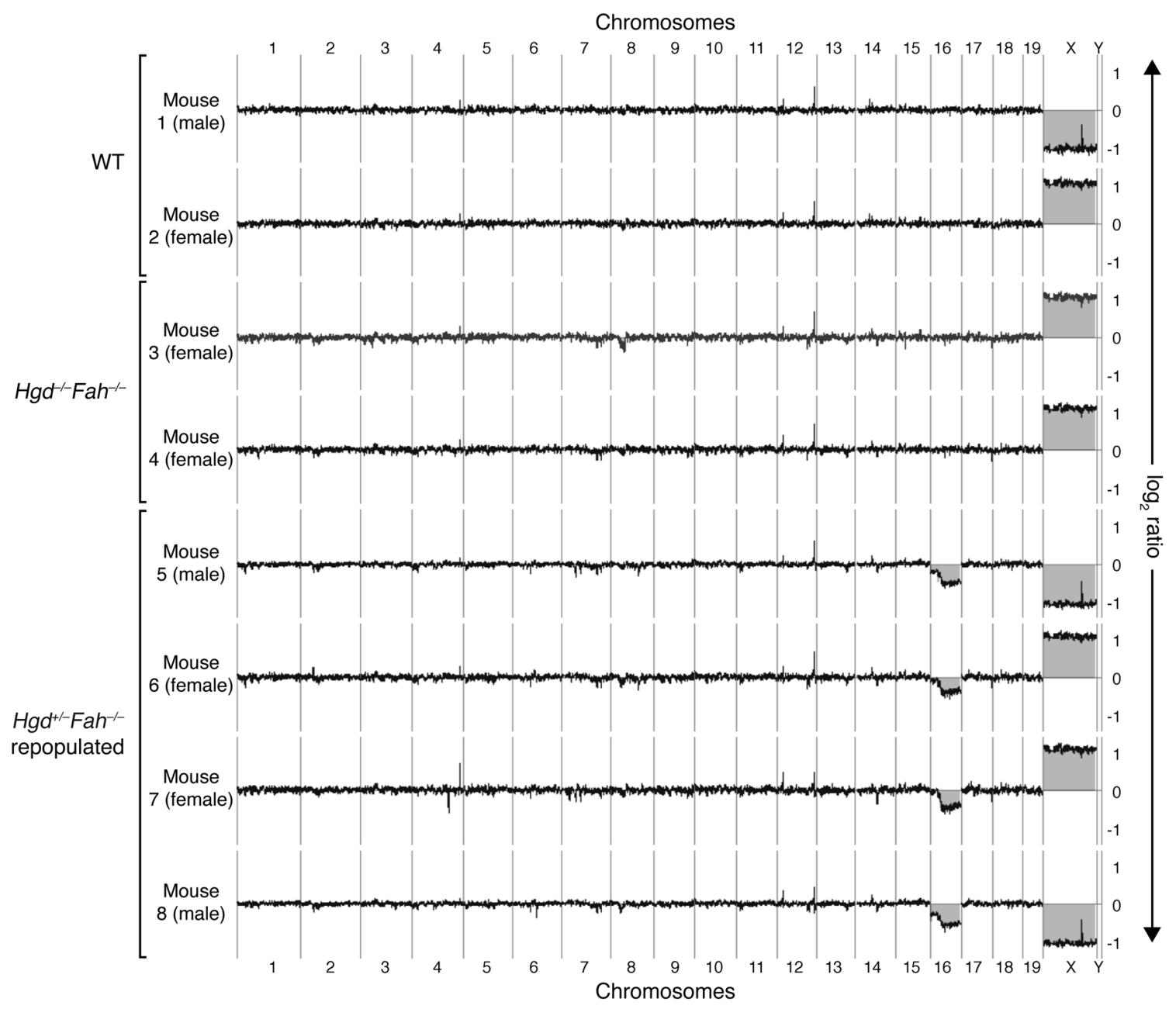

Figure 3

Copy number variation in pools of hepatocytes. Alterations in chromosome copy number were assessed by aCGH analysis using populations enriched for hepatocytes isolated from WT mice $(n=2), \mathrm{Hgd}^{-1-} \mathrm{Fah}^{-1-}$ mice off NTBC $(n=2)$ and highly repopulated $\mathrm{Hgd}^{+--} \mathrm{Fa} h^{-/-}$mice off NTBC $(n=4)$. Hybridization intensity for hepatic chromosomes is plotted as $\log _{2}$ ratio versus sex-mismatched diploid chromosomes (derived from splenocytes). The $\log _{2}$ of -1 indicates chromosome loss (e.g., loss of the $X$ chromosome in mouse 1), whereas $\log _{2}$ of 1 indicates chromosome gain (e.g., gain of the $\mathrm{X}$ chromosome in mouse 2). Copy number changes in the $\mathrm{Y}$ chromosome due to gender mismatch were detected but not shown in the plots.

chromosome-specific gains (12\%, range 5\%-20\%) and losses $(19 \%$, range $5 \%-50 \%$ ) was slightly elevated (Figure $2 \mathrm{~B}$ ), which is consistent with increased aneuploidy following extensive repopulation (15). Strikingly, chromosome 16 loss in repopulated $\mathrm{Hgd}^{+/-} \mathrm{Fab}^{-/-}$ mice was approximately 2 - to 3 -fold higher than that of any other autosome (Figure 2B). To exclude the possibility that the high degree of chromosome 16 aneuploidy was simply a consequence of Fab deficiency, hepatocytes from $\mathrm{Fah}^{-1-}$ mice off NTBC for 6 to 9 weeks were karyotyped (Figure $1 \mathrm{C}$ ). Chromosome gains and losses were equivalent to frequencies observed in adult/senior WT mice, indicating that Fab deficiency alone does not promote chromosome abnormalities. Overall, nearly half of hepatocytes from repopulated $\mathrm{Hgd}^{+/-} \mathrm{Fah}^{-/-}$mice displayed chromosome 16 aneuploidy, which was significantly higher than the degree of chromosome 16 aneuploidy observed in WT or $\mathrm{Fab}^{-/-}$mice (Figure 1D). The strong enrichment for specific loss of chromosome 16 indicates that aneuploidy contributes to hepatic functional adaptation in chronically injured $\mathrm{Hgd}^{+/-} \mathrm{Fah}^{-/-}$mice.
Genomic analysis of injury-resistant hepatocytes. A major advantage of metaphase karyotyping is elucidation of chromosome identity and copy number at the single cell level. One of the disadvantages, however, is interrogation of a relatively small sample size; typically, 20 karyotypes are determined per sample. Therefore, we performed array comparative genomic hybridization (aCGH) to analyze copy number variation from thousands of cells. This approach also enables high resolution mapping of chromosomal changes. Populations of liver cells enriched for hepatocytes were isolated from nonrepopulated and repopulated mice. Both females and males were used. Hepatic DNA was mixed with sex-mismatched reference DNA derived from WT splenocytes and subsequently hybridized to aCGH microarrays containing approximately 180,000 probes evenly dispersed throughout the genome (Supplemental Table 1). Variations in chromosome copy number are presented as a $\log _{2}$ ratio (Figure 3). A ratio of 0 indicates equivalent relative copy number between the hepatic and reference DNA. A ratio of -1 or 1 indicates loss or gain, respectively, within the entire popula- 


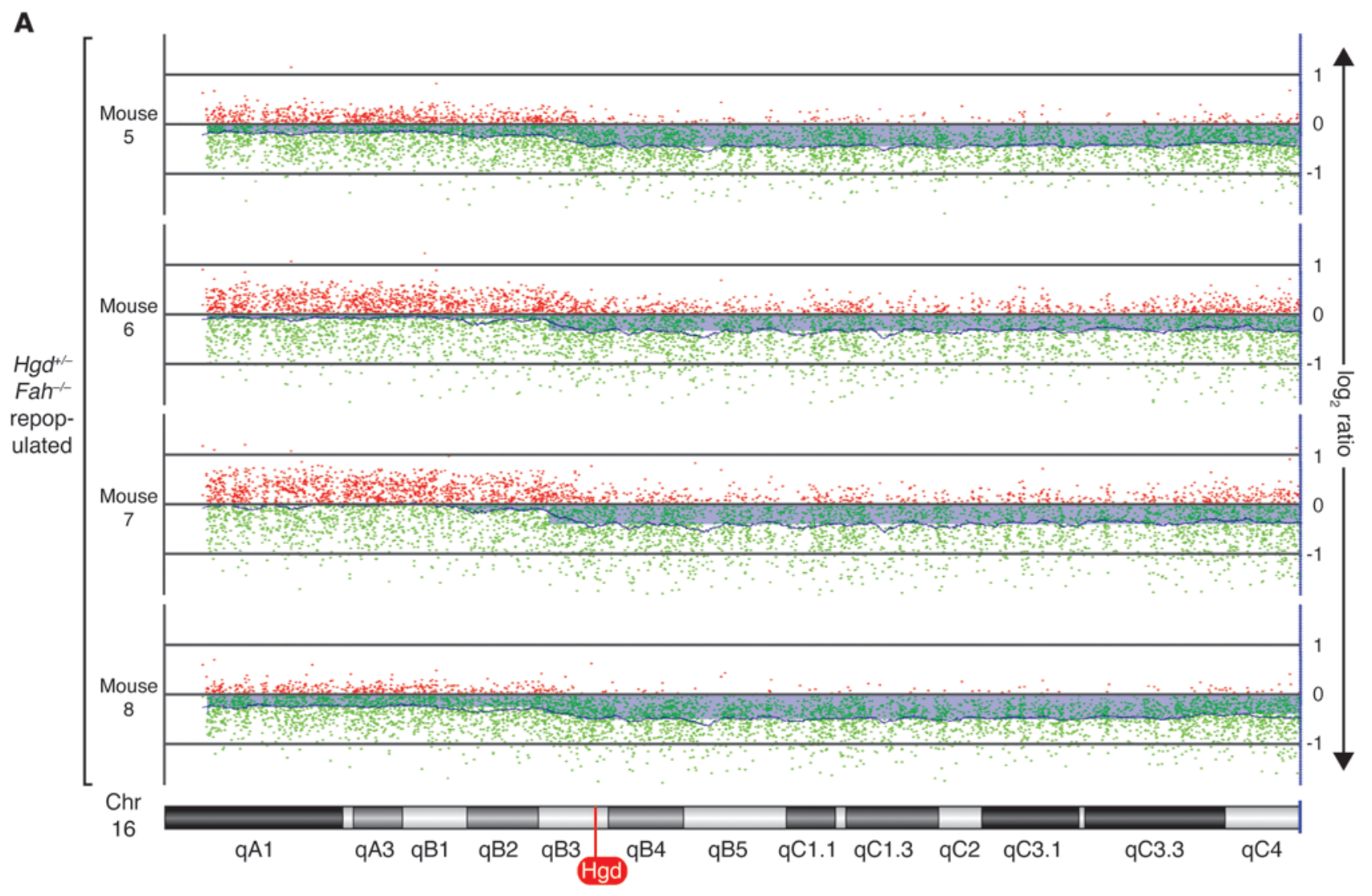

B

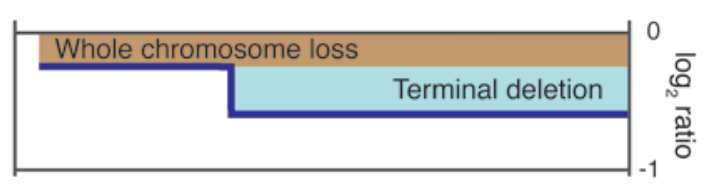

C

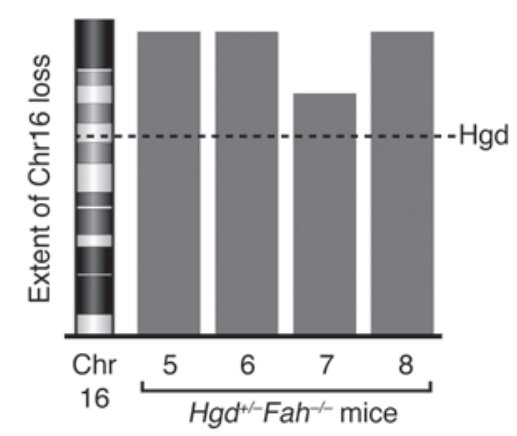

\section{Figure 4}

Loss of chromosome 16 in injury-resistant livers from $\mathrm{Hgd}^{+/-} \mathrm{Fah}^{-/-}$mice. (A) Copy number variation from aCGH analysis is shown specifically for chromosome 16. Hybridization intensity for hepatic chromosomes is plotted as $\log _{2}$ ratio versus sex-mismatched diploid chromosomes derived from splenocytes. Green dots indicate $\log _{2}$ ratio of less than 0 , and red dots indicate $\log _{2}$ ratio of more than 0 . The extent of chromosome loss is indicated along each plot (shaded purple). (B) Illustration of mosaicism for copy number loss in a heterogeneous population of cells as detected by aCGH. Whole chromosome loss and terminal deletion events are indicated. (C) Summary showing the maximum extent of chromosome 16 loss, which includes the Hgd locus.

tion of hepatic DNA. For instance, copy number variation was not detected for autosomes in mouse 1 (WT, male); however, "loss" of the $\mathrm{X}$ chromosome was reported because of the sex-mismatch hybridization (Figure 3).

Copy number variation was determined using hepatocyte genomic DNA purified from WT mice, $\mathrm{Hgd}^{-/-} \mathrm{Fah}^{-/-}$mice (off $\mathrm{NTBC}$ ), and injury-resistant $\mathrm{Hgd}^{+/-} \mathrm{Fab}^{-/-}$mice. First, analysis of WT mice revealed no changes in hepatic chromosome number among the autosomes (Figure 3; mice 1,2). The threshold for reliably detecting whole chromosome aneuploidy by aCGH is approximately $30 \%$ mosaicism. In other words, in a heterogeneous population, whole chromosome aneuploidy is only reliably detected when present in $30 \%$ or more of the cells. This limited sensitivity explains why heterogeneous chromosomespecific aneuploidy observed by karyotyping (Figure 2A), which affects chromosomes at less than $15 \%$, is undetectable by aCGH. Secondly, $\mathrm{Hgd}^{-/-} \mathrm{Fah}^{-/-}$mice were maintained without NTBC for 3 months prior to DNA isolation. Livers from these mice were refractory to tyrosinemia (Figure 1C); therefore, a dominant aneuploid karyotype was not expected. As anticipated, copy number 


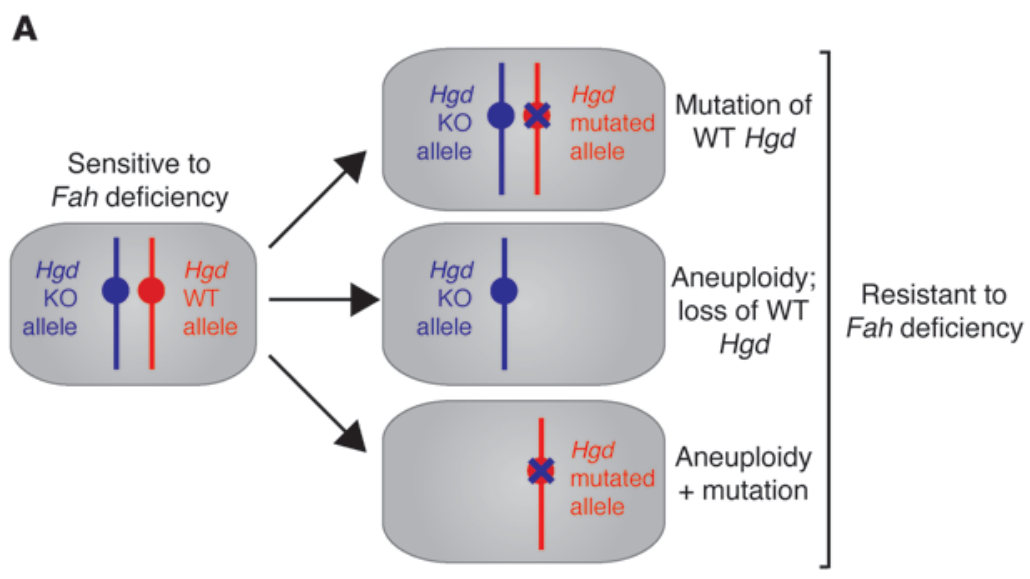

B

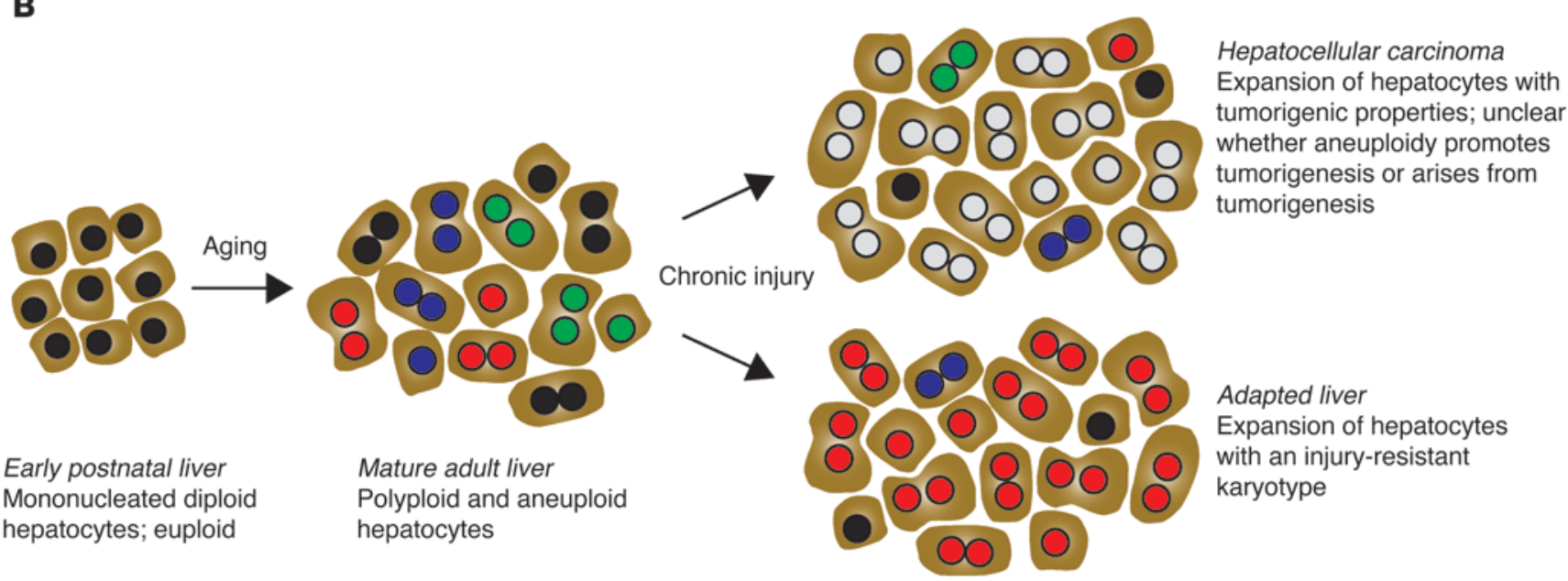

Figure 5

Models of aneuploidy-mediated adaptation and hepatocyte expansion. (A) Mechanism for adaptation to tryosinemia in $\mathrm{Hgd}^{+/-} \mathrm{Fah}{ }^{-/-}$mice. $\mathrm{Hgd}$ heterozygotes have KO and WT alleles. Loss of WT Hgd promotes resistance to Fah deficiency and occurs by mutation of the WT allele, aneuploidy (i.e., loss chromosome 16 with the WT gene), or a combination of mutation and aneuploidy. Drawings represent diploid Fah-/- hepatocytes and illustrate chromosome 16 copy number and $\mathrm{Hgd}$ status. (B) Model showing hepatocyte adaptation in response to chronic liver injury. Early in life, livers are primarily diploid and have the expected numbers of chromosomes (single black nuclei). During aging, hepatocytes polyploidize (binucleated cells) and become aneuploid (red, blue, green nuclei). Random aneuploidy affects nearly half of hepatocytes in mice and humans. Chronic liver injury can have multiple effects leading to either hepatocellular carcinoma (cells with white nuclei) or expansion injury-resistant hepatocytes (cells with red nuclei).

variation within autosomes was not seen for these mice (Figure 3; mice 3,4$)$. This result also proves that there is no specific loss of chromosome 16 associated with this genotype. Finally, aCGH analysis of hepatocytes from $\mathrm{Hgd}^{+/-} \mathrm{Fah}^{-/-}$mice, which were highly repopulated by revertant hepatocytes $(275 \%)$ and resistant to injury, revealed a single dominant autosomal alteration. Mosaicism for copy number loss involving chromosome 16 was detected in each sample (Figure 3; mice 5-8). Copy number variations were not observed in any other chromosomes.

Detailed analysis of chromosome 16 revealed 2 distinct types of aneuploidy in hepatocytes from injury-resistant $\mathrm{Hgd}^{+/-} \mathrm{Fab}^{-/-}$ mice: terminal deletion and whole chromosome loss (Figure 4, A and $\mathrm{B}$ ). Pools of hepatocytes contained a terminal deletion with a breakpoint in the $\mathrm{qB} 2$ region of chromosome 16 (Figure 4A; mice $5-8)$. The $\log _{2}$ ratios in the deleted region were -0.33 to -0.40 , indicating terminal deletion in $40 \%-50 \%$ of the cells from these mice. Importantly, whole chromosome loss was detected in 3 of 4 samples (Figure 4A; mice 5, 6, 8). The $\log _{2}$ ratios for the proximal segment of chromosome 16 were between 0 and -0.25 , indicating whole chromosome loss in less than $30 \%$ of hepatocytes. Failure to detect the deletion event by karyotype analysis or to differentiate whole chromosome loss and rearrangement leading to the deletion reflects a limitation of the metaphase cytogenetic approach. Karyotypes are only available for cells that reach metaphase following short-term expansion in vitro, and the chromosome makeup of nonproliferating cells is not determined. Identification of both whole chromosome loss and deletion events in heterogeneous populations of cells is a major advantage of aCGH technology.

Hepatic aneuploidy to date has been characterized in terms of gain and loss of whole chromosomes $(12,17)$. Deletion of the terminal region of chromosome 16 in hepatocytes from these mice is the first deletion identified by our group. We speculate that the mutagenic effects resulting from Fab deficiency contributed to 
chromosome 16 breakage (24). Nevertheless, $H g d$ was lost in cases of whole chromosome loss and terminal deletion (Figure 4C). Thus, similar to our karyotyping experiments, hepatocytes monosomic for chromosome 16 made up a substantial fraction of livers from injury-resistant $\mathrm{Hgd}^{+/-} \mathrm{Fab}^{-/-}$mice.

\section{Discussion}

A vast body of literature on the detrimental effects of aneuploidy exists. During human development, chromosomal imbalances almost always cause birth defects (reviewed in refs. 25, 26). Similarly, chromosome instability (CIN) has long been thought to be a cause of cancer (27). For this reason, it came as a surprise to find that a highly regenerative tissue such as the liver contains a large percentage of aneuploid cells $(12,15,17)$, even in very young individuals. Since liver cancer is rare in children, the pervasive presence of aneuploid hepatocytes challenges the notion of a causal connection between aneuploidy and cancer. The finding furthermore raises a very different question: can aneuploidy be beneficial and proadaptive? Recent studies in budding yeast strongly suggest that aneuploidy, in fact, can greatly accelerate adaptive evolution $(18,19)$.

To test whether adaptive evolution occurs in the liver, we utilized the $\mathrm{Fah}^{-/-}$model of hereditary tyrosinemia (Figure 1A). Loss of Fab leads to accumulation of fumarylacetoacetate and associated toxic metabolites, resulting in liver failure and death. Liver function can be maintained by blocking the pathway upstream of FAH by treatment with NTBC or loss of HGD. Previous studies showed that when $\mathrm{Hgd}^{+/-} \mathrm{Fab}^{-/-}$mice were challenged by NTBC withdrawal, revertant healthy nodules rapidly emerged with the capacity to repopulate the entire liver (21). Furthermore, approximately $25 \%$ of these nodules contained spontaneous mutations in $\mathrm{Hgd}$, effectively rendering the nodules $H g d$ null (Figure 5A). Here, we showed that aneuploidy also promotes adaptation to tyrosinemia-induced liver stress. Livers repopulated with reversion hepatocytes were markedly enriched for chromosome 16 aneuploidy (e.g., either whole chromosome loss or terminal deletion). In $\mathrm{Hgd}^{+/-} \mathrm{Fah}^{-/-}$mice, loss of chromosome 16 containing functional $\mathrm{Hgd}$ leads to complete loss of HGD activity, producing hepatocytes resistant to tyrosinemia (Figure 5A). It is important to emphasize the rapid kinetics of this phenomenon: $H g d$-deficient revertant nodules are seen at the earliest time points after NTBC withdrawal. This is consistent with our previous observations that aneuploidy is present in healthy liver. Thus, the loss of chromosome 16 is not caused by the injury, but simply reflects selection/expansion of preexisting injury-resistant hepatocytes.

Karyotypic adaptive evolution could be a common mechanism in many human liver diseases. Recent work from our group showed that approximately half of hepatocytes from healthy human donors were aneuploid, and each chromosome was equally affected (17). Thus, selective proliferation by subsets of aneuploid hepatocytes could yield divergent effects (Figure 5B). On the one hand, hepatic aneuploidy could have pathological consequences. Aneuploidy has been extensively described in hepatocellular carcinoma (28-30), but it is unknown whether tumors arose from preexisting aneuploid hepatocytes or simply became aneuploid during tumorigenesis. It is intriguing that whole chromosome gains/losses were reported in dozens of lesions (28). On the other hand, preexisting aneuploid hepatocytes could have beneficial effects, such as adapta- tion to chronic liver injury, just as we demonstrated with the $\mathrm{Hgd}^{+/-} \mathrm{Fah}^{-/-}$model. Indeed, studies have shown that chronic liver damage induced by hepatitis $\mathrm{C}$ infection gave rise to nonmalignant nodules of liver tissue (31-33). Analysis of $X$ inactivation in tissue from female patients revealed up to $50 \%$ of these nodules were clonal, indicating expansion from a single injury-resistant cell (32). Experiments are underway to elucidate the extent to which hepatic aneuploidy contributes to stress-induced liver adaptation in humans.

Note that mouse and human chromosomes are nonsyntenic, indicating that loci on a given chromosome in one species are not always linked in the other species (34). For instance, $\mathrm{Hgd}$ is found on chromosome 16 in mice and chromosome 3 in humans. Therefore, caution must be observed when interpreting the functional impact of chromosome-specific aneuploidy between species.

In conclusion, although clonal aneuploidy in cirrhosis has traditionally been interpreted as a premalignant event, our results indicate that this may not necessarily be the case. Specific gains and losses of chromosomes harboring injury-resistance alleles in normal, nontransformed hepatocytes may render them differentially resistant to chronic insults such as viral hepatitis as well as alcohol- and fat-induced hepatitis. We propose that it is time to revisit the question of clonal evolution in human liver cirrhosis with an eye toward proadaptive and protective aneuploidy. Perhaps aCGH will become a diagnostic and prognostic tool for hepatologists in the future, as it is in oncology today.

\section{Methods}

Mouse strains and animal husbandry. Inbred mouse strains were used: WT (C57BL/6, 129S4), transgenic Rosa26-lacZ (C57BL/6 and 129S4) (35), $\mathrm{Fah}^{-/-}$(C57BL/6 and 129S4) (36), and $\mathrm{Hgd}^{-/-}(37,38) . \mathrm{Fab}^{-/}, \mathrm{Hgd} d^{+-}$and transgenic Rosa26-lac $Z$ mice were crossed to obtain the desired genotypes. All mutant animals were maintained on NTBC ( $4 \mathrm{mg} / \mathrm{l})$ until age 3 to 4 months, at which time NTBC was removed. Livers from $\mathrm{Hgd} d^{+/-} \mathrm{Fah}^{-/-}$mice were harvested following either 3 to 4 weeks or 2 to 3 months of NTBC withdrawal. Livers from $\mathrm{Fah}^{-/-}$mice were harvested after 2 to 3 sessions of NTBC withdrawal of 3 weeks per session.

Cell isolation. Primary hepatocytes were isolated by 2-step collagenase perfusion (23). For karyotype studies, cell suspensions were washed 2 times in DMEM/F12 (Invitrogen) and 0.5\% FBS (Hyclone) using low-speed centrifugation $(50 \mathrm{~g} \times 1$ minute) to enrich for hepatocytes, which are larger and heavier than nonparenchymal cells. Additional hepatocyte purification was performed for aCGH experiments. Populations of cells enriched for hepatocytes were isolated with an InFlux flow cytometer (BD) using a $150-\mu \mathrm{m}$ nozzle. Cells adhering to each other (i.e., doublets) were eliminated on the basis of pulse width. Nonparenchymal cells were excluded based on expression of CD45 (APC), Mac-1 (PE), and CD133 (APC) using antibodies from eBioscience. Dead cells were excluded on the basis of $5 \mu \mathrm{g} / \mathrm{ml}$ propidium iodide (Invitrogen) incorporation. Hepatocyte populations were gated according to size and granularity. Splenocytes were isolated from 2-month-old C57BL/6 mice (male and female); erythrocytes were removed using RBC Lysis Buffer (eBioscience).

Histology and immunocytochemistry. Histological analyses (39) and FAH immunocytochemistry (15) were previously reported. Images of whole tissue sections were stitched together using AxioVision 4.7 software (Carl Zeiss) from individual tiles acquired on a Zeiss MRc color camera mounted on a Zeiss AxioObserver with motorized stage control.

Karyotypes. First, freshly isolated primary hepatocytes were seeded at 5,000-7,500 cells $/ \mathrm{cm}^{2}$ on Primaria tissue culture plastic (BD) and incu- 
bated in DMEM/F12 (Invitrogen), 0.5\% FBS (Hyclone), ITS Supplement (contains $5 \mu \mathrm{g} / \mathrm{ml}$ insulin, $5 \mu \mathrm{g} / \mathrm{ml}$ transferrin and $5 \mathrm{ng} / \mathrm{ml}$ sodium selenite; Invitrogen), $15 \mathrm{mM}$ HEPES, and antibiotic-antimycotic (Cellgro). After 4 hours, cells were washed once with DMEM/F12 to remove nonadherent cells and provided fresh culture medium supplemented with $50 \mathrm{ng} / \mathrm{ml}$ human epidermal growth factor (Invitrogen) and $1.0 \mu \mathrm{M}$ 3,3',5-Triiodo-L-thyronine sodium salt $\left(\mathrm{T}_{3}\right)$ (Sigma-Aldrich). Second, after approximately 40 hours, hepatocytes were treated with $30 \mathrm{ng} / \mathrm{ml}$ KaryoMax Colcemid Solution (Invitrogen) for 3 hours and harvested by trypsinization. Third, after extensive washing, cells were incubated for 15 minutes in $75 \mathrm{mM} \mathrm{KCl}$ and fixed with methanol/acetic acid (3:1 ratio). Finally, chromosomes from approximately 20 metaphasearrested hepatocytes per sample were G-banded with a standard trypsin/Wright's stain protocol. Photographs were taken using CytoVision software from Applied Imaging.

Array CGH. Genomic DNA from hepatocyte and splenocyte samples was isolated using DNeasy Blood and Tissue Kit (QIAGEN). Array CGH was performed using Agilent 180K SurePrint G3 Mouse Microarray (cat. no. G4826A-027411, NCBI Build 37) with $1.8 \mathrm{~kb}$ overall median probe spacing. DNA digestion, labeling, and hybridization were performed according to the manufacturer's instructions, with slight modifications as described (40). Briefly, $1 \mu \mathrm{g}$ of genomic DNA from test (i.e., hepatocytes) and $1 \mu \mathrm{g}$ of reference (i.e., splenocytes) samples were digested with $A l u \mathrm{I}$ and $R s a \mathrm{I}$ (Promega) at $37^{\circ} \mathrm{C}$ for 2 hours and labeled by cyanine 5-dCTP (for the test sample) or cyanine 3-dCTP (for the reference sample) (PerkinElmer) at $37^{\circ} \mathrm{C}$ for 2 hours using the Bioprime CGH Labeling Module (Invitrogen). Labeled DNA of the test samples and gender-mismatched Microarray image files were quantified using Agilent Feature Extraction software (version 9.5), and text file outputs were imported into Agilent Genomic Workbench software (version 6.5). To identify chromosomal aneuploidy and genomic copy number variations, data were analyzed using the Aberration Detection Method 2 (ADM2) statistical algorithm, which uses an iterative procedure to identify all genomic regions for which the weighted average by $\log _{2}$ ratio of the measured probe signals is different from the expected value of 0 by more than a given threshold. "Fuzzy Zero" and "Centralization" default algorithms of DNA Analytics were utilized. To minimize the detection of nonrelevant variants, data analyses were performed using threshold 6.0 and changes detected by less than 150 probes $(<300 \mathrm{~kb})$ were filtered. Data were also analyzed using ADM2 with threshold 3.0 and using algorithm ADM1 with or without filters, which detected no additional low-level mosaicism of aneuploidy. Raw data files (GEO GSE38963) are available via GEO.

Statistics. Statistical significance (Figure 2D) was determined using 2 -sided Student's $t$ test. $P$ values of less than 0.05 were considered significant. Statistical analysis of aCGH data was described above.

Study approval. The Institutional Animal Care and Use Committee of Oregon Health and Science University approved all mouse experiments.

\section{Acknowledgments}

We thank S. Kaech Petrie (Advanced Light Microscopy Core at Oregon Health and Science University, core grant S10-RR023432), G. Banker for use of the Zeiss AxioObserver microscope, and A. Major (Morphology Core of the Texas Medical Center, grant DK56338) for histology support. We are grateful to J. Coleman, A. Haft, and S. Nygaard for expert management of the mouse colony and the laboratory. This work was supported by grants from the NIH to M. Grompe (DK048252) and A.W. Duncan (F32DK076232).

Received for publication March 27, 2012, and accepted in revised form June 28, 2012.

Address correspondence to: Andrew W. Duncan, Department of Pathology, McGowan Institute for Regenerative Medicine, University of Pittsburgh, 450 Technology Drive, Suite 300, Pittsburgh, Pennsylvania 15219, USA. Phone: 412.624.5302; Fax: 412.624.5363; E-mail: duncana@pitt.edu.
1. Tujios S, Fontana RJ. Mechanisms of drug-induced liver injury: from bedside to bench. Nat Rev Gastroenterol Hepatol. 2011;8(4):202-211.

2. Smith BW, Adams LA. Non-alcoholic fatty liver disease. Crit Rev Clin Lab Sci. 2011;48(3):97-113.

3. Stocker E, Wullstein HK, Brau G. Capacity of regeneration in liver epithelia of juvenile, repeated partially hepatectomized rats. Virchows Arch B Cell Pathol. 1973;14(2):93-103.

4. Overturf K, Al-Dhalimy M, Ou CN, Finegold M, Grompe M. Serial transplantation reveals the stem-cell-like regenerative potential of adult mouse hepatocytes. Am J Pathol. 1997;151(5):1273-1280.

5. Kudryavtsev BN, Kudryavtseva MV, Sakuta GA, Stein GI. Human hepatocyte polyploidization kinetics in the course of life cycle. Virchows Arch B Cell Pathol Incl Mol Pathol. 1993;64(6):387-393.

6. Toyoda H, et al. Changes to hepatocyte ploidy and binuclearity profiles during human chronic viral hepatitis. Gut. 2005;54(2):297-302.

7. Guidotti JE, Bregerie O, Robert A, Debey P, Brechot C, Desdouets C. Liver cell polyploidization: a pivotal role for binuclear hepatocytes. J Biol Chem. 2003; 278(21):19095-19101.

8. Faktor VM, Uryvaeva IV. Progressive polyploidy in mouse liver following repeated hepatectomy. Tsitologiia. 1975;17(8):909-916.

9. Yim AP. Some flow-cytofluorimetric studies of the nuclear ploidy of mouse hepatocytes: iii. Br J Exp Pathol. 1982;63(4):458-461.

10. Margall-Ducos G, Celton-Morizur S, Couton D, Bregerie $\mathrm{O}$, Desdouets C. Liver tetraploidization is controlled by a new process of incomplete cytokinesis.
J Cell Sci. 2007;120(pt 20):3633-3639.

11. Wang $X$, et al. Cell fusion is the principal source of bone-marrow-derived hepatocytes. Nature. 2003; 422(6934):897-901

12. Duncan AW, et al. The ploidy conveyor of mature hepatocytes as a source of genetic variation. Nature. 2010;467(7316):707-710.

13. Overturf K, Al-Dhalimy M, Finegold M, Grompe M. The repopulation potential of hepatocyte populations differing in size and prior mitotic expansion. Am J Pathol. 1999;155(6):2135-2143.

14. Weglarz TC, Degen JL, Sandgren EP. Hepatocyte transplantation into diseased mouse liver. Am J Pathol. 2000;157(6):1963-1974.

15. Duncan AW, et al. Ploidy reductions in murine fusion-derived hepatocytes. PLoS Genet. 2009; 5(2):e1000385.

16. Ganem NJ, Godinho SA, Pellman D. A mechanism linking extra centrosomes to chromosomal instability. Nature. 2009;460(7252):278-282.

17. Duncan AW, et al. Frequent aneuploidy among normal human hepatocytes. Gastroenterology. 2012; 142(1):25-28.

18. Pavelka N, et al. Aneuploidy confers quantitative proteome changes and phenotypic variation in budding yeast. Nature. 2010;468(7321):321-325.

19. Rancati G, et al. Aneuploidy underlies rapid adaptive evolution of yeast cells deprived of a conserved cytokinesis motor. Cell. 2008;135(5):879-893.

20. Lindblad B, Lindstedt S, Steen G. On the enzymic defects in hereditary tyrosinemia. Proc Natl Acad Sci US A. 1977;74(10):4641-4645.

21. Manning K, Al-Dhalimy M, Finegold M, Grompe M.
In vivo suppressor mutations correct a murine model of hereditary tyrosinemia type I. Proc Natl Acad SciU S A. 1999;96(21):11928-11933.

22. Endo F, et al. Complete rescue of lethal albino c14CoS mice by null mutation of 4-hydroxyphenylpyruvate dioxygenase and induction of apoptosis of hepatocytes in these mice by in vivo retrieval of the tyrosine catabolic pathway. J Biol Chem. 1997; 272(39):24426-24432.

23. Overturf $K$, et al. Hepatocytes corrected by gene therapy are selected in vivo in a murine model of hereditary tyrosinaemia type I. Nat Genet. 1996; 12(3):266-273.

24. Jorquera R, Tanguay RM. The mutagenicity of the tyrosine metabolite, fumarylacetoacetate, is enhanced by glutathione depletion. Biochem Biophys Res Commun. 1997;232(1):42-48.

25. Antonarakis SE, Lyle R, Dermitzakis ET, Reymond A, Deutsch S. Chromosome 21 and down syndrome: from genomics to pathophysiology. Nat Rev Genet. 2004;5(10):725-738.

26. Leppig KA, Disteche CM. Ring X and other structural X chromosome abnormalities: $\mathrm{X}$ inactivation and phenotype. Semin Reprod Med. 2001; 19(2):147-157

27. Gordon DJ, Resio B, Pellman D. Causes and consequences of aneuploidy in cancer. Nat Rev Genet. 2012; 13(3):189-203.

28. Kim TM, et al. Clinical implication of recurrent copy number alterations in hepatocellular carcinoma and putative oncogenes in recurrent gains on 1q. Int J Cancer. 2008;123(12):2808-2815.

29. Nalesnik MA, et al. Gene deletions and amplifica- 
tions in human hepatocellular carcinomas: correlation with hepatocyte growth regulation. $A m J$ Pathol. 2012;180(4):1495-1508.

30. Fu L, et al. Down-regulation of tyrosine aminotransferase at a frequently deleted region $16 \mathrm{q} 22$ contributes to the pathogenesis of hepatocellular carcinoma. Hepatology. 2010;51(5):1624-1634.

31. Ochiai T, Urata Y, Yamano T, Yamagishi H, Ashihara $\mathrm{T}$. Clonal expansion in evolution of chronic hepatitis to hepatocellular carcinoma as seen at an X-chromosome locus. Hepatology. 2000;31(3):615-621.

32. Paradis $\mathrm{V}$, et al. Clonal analysis of micronodules in virus $\mathrm{C}$-induced liver cirrhosis using laser capture microdissection (LCM) and HUMARA assay. Lab Invest. 2000;80(10):1553-1559.
33. Paradis V, Laurendeau I, Vidaud M, Bedossa P. Clonal analysis of macronodules in cirrhosis. Hepatology. 1998;28(4):953-958.

34. Carver EA, Stubbs L. Zooming in on the humanmouse comparative map: genome conservation reexamined on a high-resolution scale. Genome Res. 1997;7(12):1123-1137.

35. Friedrich G, Soriano P. Promoter traps in embryonic stem cells: a genetic screen to identify and mutate developmental genes in mice. Genes Dev. 1991; 5(9):1513-1523.

36. Grompe $M$, et al. Loss of fumarylacetoacetate hydrolase is responsible for the neonatal hepatic dysfunction phenotype of lethal albino mice. Genes Dev. 1993;7(12A):2298-2307.
37. Manning K, Fernandez-Canon JM, Montagutelli $\mathrm{X}$, Grompe M. Identification of the mutation in the alkaptonuria mouse model. Hum Mutat. 1999; 13(2): 171.

38. Montagutelli X, Lalouette A, Coude M, Kamoun P, Forest M, Guenet JL. aku, a mutation of the mouse homologous to human alkaptonuria, maps to chromosome 16. Genomics. 1994;19(1):9-11.

39. Overturf K, et al. Adenovirus-mediated gene therapy in a mouse model of hereditary tyrosinemia type I. Hum Gene Ther. 1997;8(5):513-521.

40. Ou Z, et al. Bacterial artificial chromosome-emulation oligonucleotide arrays for targeted clinical array-comparative genomic hybridization analyses. Genet Med. 2008;10(4):278-289. 